\title{
Numerical simulation of an inner engaging gerotor based on the optimization of inlet and outlet cavities
}

\author{
${ }^{*}$ Xiaohu Sang ${ }^{1 \mathrm{a}}$, Xiaojun Zhou ${ }^{1 \mathrm{~b}}$, Xiaoguang Liu ${ }^{1 \mathrm{c}}$ \\ ${ }^{1}$ School of Mechatronics Engineering and Automation,Shanghai University,Shanghai,China \\ ”asangxh7@qq.com, bsdzhouxj@shu.edu.cn, 'xiaoguangvisa@126.com
}

\begin{abstract}
Keywords: Gerotor pump; Inlet and outlet cavities; Optimization design; Pumplinx
Abstract. Based on the available theoretical and half empirical methods of gerotor pump design, the optimization of inlet and outlet cavities for an inner engaging ellipse gerotor has been presented. The interior flow inside of the optimized cavities and pump has been observed by using software Pumplinx, and the results show that the optimized inlet and outlet cavities have a higher volumetric efficiency. Then, the numerical investigation of the gerotor has been carried out with the commercial software Pumplinx. The simulation results show a good agreement with the theoretical data, the correctness of the simulation results was verified. The pressure is uniform in most area of the inlet and outlet cavity and cavitation phenomenon has not been detected. It provides guide for the oil pump design.
\end{abstract}

\section{Introduction}

Internal cycloid gerotor pump has the merits of small size, light weight, strong suction, low noise, small flow pulsation etc, and it is widely used in small and medium power engines.

In domestic, the main studies on internal cycloid gerotor pump is base on the available theoretical and half empirical methods, such as Huayong Mao ${ }^{[1]}$, deduced the area equation of the inlet and outlet cavities, and analyzed the influence of rev, oil viscosity and the way of oil inlet. Shengguan Qu ${ }^{[2]}$, analyzed the reason of volumetric efficiency loss, and put forward some measures like, chose the appropriate inlet and outlet diameter to enhance the volumetric efficiency. In west, R. Elayaraja ${ }^{[3]}$, used the CFD software STAR-CD simulated the interior flow, and gave the relationship of the periodical flux pulsation.

In the article, we use the available theoretical and half empirical methods to optimized the inlet and outlet cavities and give the equations of cavities designing, and find out the best solution, then use the software Pumplinx to analyse the interior flow inside of the optimized cavities and pump, The simulation results show a good agreement with the theoretical data,the correctness of the simulation results was verified.

\section{Optimization of cavities}

The oil pump is made up of cavities, inner and outer rotor, groove, inlet and outlet. The outer rotor has one more tooth than the inner rotor. The crescent shaped cavities are fixed to the pump body or pump cover, the inner edge of the crescent shaped cavities are the circle with center at $O_{l}$ and radius equal to the radius of the dedendum circle ${ }^{[4]}$, the outer edge of the crescent shaped cavities are the circle with center at $\mathrm{O}_{2}$ and radius equal to the radius of the dedendum circle too. However, the actual position of the cavities should be confirm by the the meshing point $Q$ of the inlet area and the outlet area. 


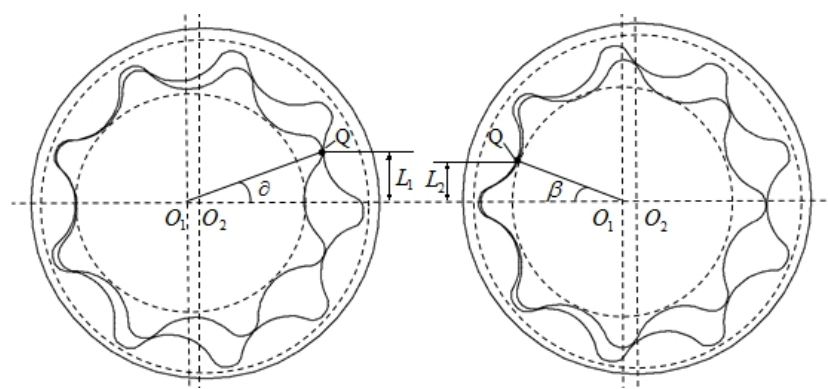

Fig.1 Schematic for the engaging

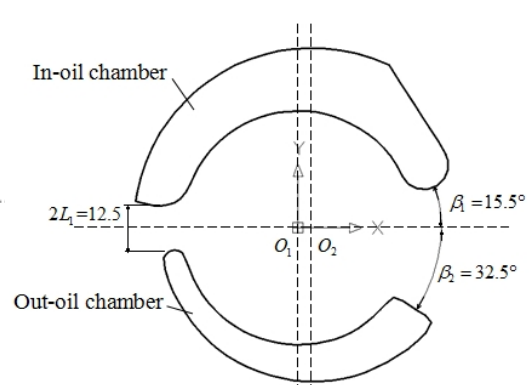

Fig.2 Schematic for the cavity structure

In the Fig. 1, we can observe when the corner of the $\varphi_{1}=\pi$, the area of the inner-rotor closed region is maximum. So, we can obtain the distance from the point $Q$ to $X$-axis:

$$
L_{1}=R_{c} \sin \varphi_{2}-r_{c} \sin \left(\varphi_{2}+\theta\right)
$$

Where, $R_{c}$ is the radius of generating circle, $r_{\mathrm{c}}$ is the radius of arc teeth circle; $\varphi_{2}=\pi z_{1} / \mathrm{z}_{2} ; k$ is the coefficient of creation; $\tan \theta=\frac{\sin \varphi_{2}}{k-\cos \varphi_{2}}$;

The included angle of the point $Q$ is :

$$
\partial=\tan ^{-1}\left(\frac{R_{c} \sin \varphi_{2}-r_{c} \sin \left(\varphi_{2}+\theta\right)}{R_{c} \cos \varphi_{2}-r_{c} \cos \left(\varphi_{2}+\theta\right)-e}\right)
$$

When the corner of the inner-rotor $\varphi_{1}=\pi / z_{1}$, the area of the closed region is minimum.Substituting $\varphi_{2}=\pi / z_{2}$ into Eq.(1) can get the value of the $L_{2}$ and $\beta$. The cavities in theory is exactly asymmetrical, but in order to obtain good oil absorption, we can decrease the value of the $2 L_{1}$ or magnify the degree $\beta_{1}$ and $\beta_{2}$, Fig. 2 shows the schematic for the cavity structure. Tab. 1 shows the whole Parameters of the cavities.

Tab.1 Parameters of the cavities

\begin{tabular}{|c|c|c|c|c|c|}
\hline Parameter & Value & Parameter & Value & Parameter & Value \\
\hline $\mathrm{Z}_{1}$ & 8 & $\mathrm{R}_{\mathrm{c}}(\mathrm{mm})$ & 14.55 & $\mathrm{~L}_{1}(\mathrm{~mm})$ & 6.5 \\
\hline $\mathrm{Z}_{2}$ & 9 & $\mathrm{e}(\mathrm{mm})$ & 2 & $\beta_{1}\left(^{\circ}\right)$ & $16.5^{\circ}$ \\
\hline $\mathrm{k}$ & 1.3 & $\mathrm{r}_{\mathrm{c}}(\mathrm{mm})$ & 8 & $\beta_{2}\left(^{\circ}\right)$ & $32.5^{\circ}$ \\
\hline
\end{tabular}

\section{Confirm the area of the alveoli and theoretical displacement}

The theoretical displacement of oil pump can be obtained by the Eq.(3):

$$
V=B \cdot Z_{1} \cdot\left(S_{\max }-S_{\min }\right)
$$

Where, $S_{\max }$ is the maximum tooth area; $S_{\min }$ is the minimum tooth area; $B$ is the tooth thickness . $S_{\max }$ and $S_{\min }$ can be got by using the tools planar surface-Measurement Area in SolidWorks.

Substituting the measured data in Fig.3 into Eq.(3) can get the theoretical flux of oil pump. $V=20 * 8 *(44.14-0.88)=6963.29(\mathrm{ml} / \mathrm{r})=6.9632(1 / \mathrm{r})$

And the theoretical flux can be expressed as Eq.(4) :

$Q=n * V$ ( $n$ is rotational speed)

$Q=4000 * 6.9632=27.85(\mathrm{~L} / \mathrm{min})$, the theoretical flux at the speed of $5000 \mathrm{r} / \mathrm{min}$ is $34.816 \mathrm{~L} / \mathrm{min}$. 

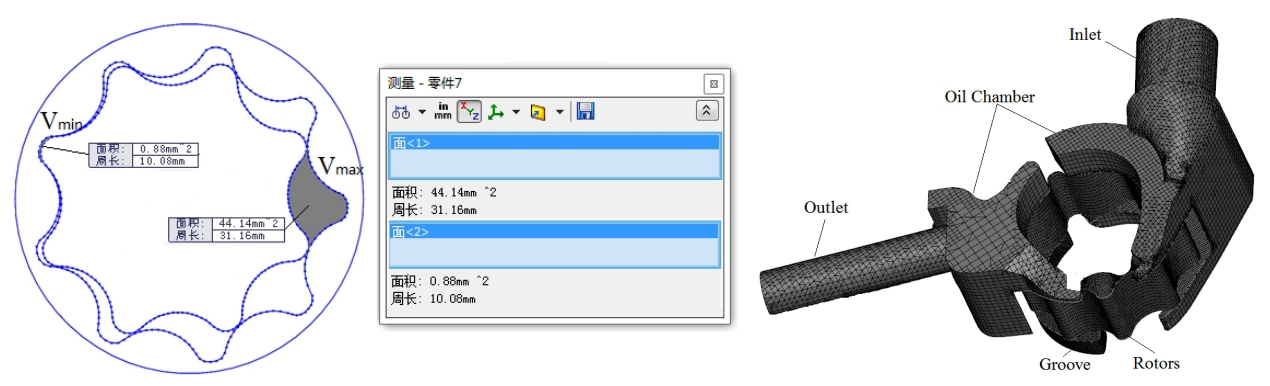

Fig. 3 The tooth area measured by SolidWorks

Fig.4 The binary tree meshes of the oil pump

\section{Numerical simulation analysis}

PumpLinx is an unique CFD tool created to help engineers design better fluid pumps \& motors, it solves more pump types than other commercial software ${ }^{[5]}$.

\section{Mesh generation and boundary condition}

Mesh the flow domain by using the embedded mesher in PumpLinx. The special binary tree mesher can create cartesian cells which has excellent orthogonal, split automatically at clearances, sharp edges etc. with thicker cells, so that the flow domain can be described with less cells but higher precision. Besides, the operation is simple, efficient and time saving. Fig. 4 shows a binary tree mesh on whole part of pump. The whole job can be finished in several minutes, and the number of cells is about 126525 .

\section{Turbulence model}

Appropriate turbulence model can describe the flow situation inside the oil pump accurately ${ }^{[6]}$. Choosing a proper turbulence model can decrease the simulation errors, and enhance the accuracy of performance prediction. Standard $k$ and RNG are the most widely adopted models for turbulence modeling in pumps. This paper uses RNG model, which can satisfy the need of oil pumps' turbulence prediction, not only in convergence, but also in accuracy.Its detailed properties are shown in Tab.2.

Tab. 2 boundary condition parameters

\begin{tabular}{|l|l|l|l|}
\hline Parameter & Value & Parameter & Value \\
\hline Pressure-inlet $(\mathrm{Pa})$ & 101325 & Oil dynamic viscosity $(\mathrm{Pa} . \mathrm{s})$ & 0.00705 \\
\hline Pressure-outlet $(\mathrm{MPa})$ & 0.4 & Oil bulk modulus $(\mathrm{Pa})$ & $1.5 \mathrm{E} 9$ \\
\hline Oil density $\left(\mathrm{kg} / \mathrm{m}^{3}\right)$ & 800 & Gas density $(\mathrm{kg} / \mathrm{m} 3)$ & 0.0245 \\
\hline Speed $(\mathrm{r} / \mathrm{min})$ & 2000 & Temperature $(\mathrm{K})$ & 300 \\
\hline Saturation pressure $(\mathrm{Pa})$ & 400 & Vapor pressure $(\mathrm{Pa})$ & 3610 \\
\hline Gas mass fraction & $2.3 \mathrm{E}-5$ & & \\
\hline
\end{tabular}

\section{Results}

\section{Flow volumetric flux}

Fig.18(a) to (e) shows the flow volumetric flux@outlet, the flow volumetric flux@outlet increased with the rotational speed increases. But decreased at the speed of $4000 \mathrm{r} / \mathrm{min}$ and become unstable. The reason for this is the pump working at a high speed, the oil becoming progressively more difficult to supply and creates a vacuum. Gas in oil will be dumped by inertial force this moment, forming many small bubbles; and the bubbles' forming, flowing and deforming lead to the cavitation. 


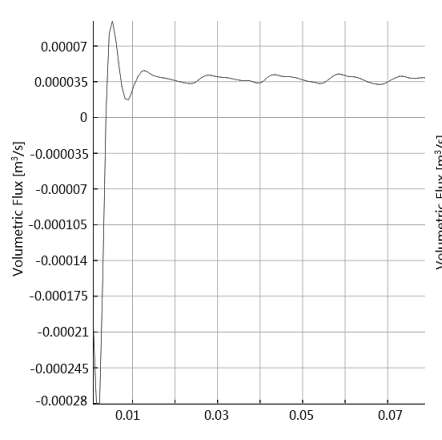

(a) $500 \mathrm{r} / \mathrm{min}$

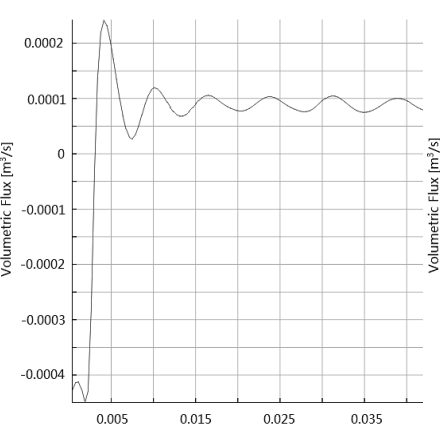

(b) $1000 \mathrm{r} / \mathrm{min}$

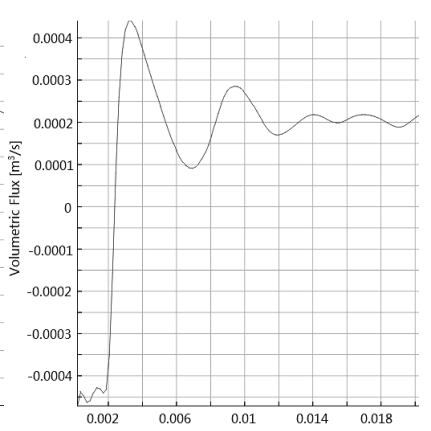

(c) $2000 \mathrm{r} / \mathrm{min}$

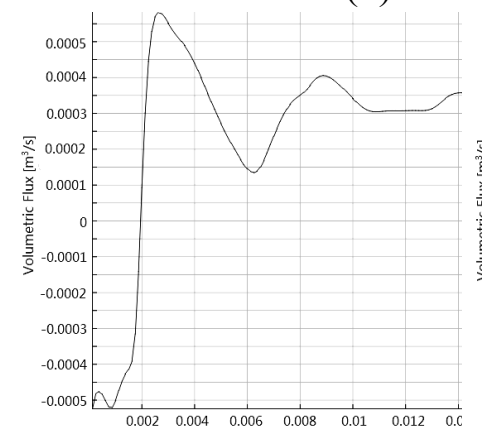

(d) $3000 \mathrm{r} / \mathrm{min}$

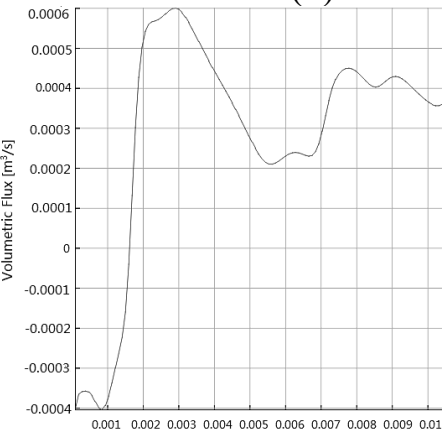

(e) $4000 \mathrm{r} / \mathrm{min}$

Fig. 5 The volumetric flux @ outlet in different speed

Besides, from the Fig.5 we also can get the approximate flux of the pump; for example, the flux at the speed of $4000 \mathrm{r} / \mathrm{min}$ can be obtained by the Eq.(5):

$$
Q=0.0004 * 60000=24(1 / \mathrm{min})
$$

According the Eq.(4) we know the theoretical flux $Q$. Certainly, the volumetric efficiency (VE) can be also got, After we get the value at every speed, the flow characteristic curve can be easy get as the Fig. 6 shows. We can observe that the CFD result is close to the theoretical; there are small differences at the high speed, but it is acceptable.

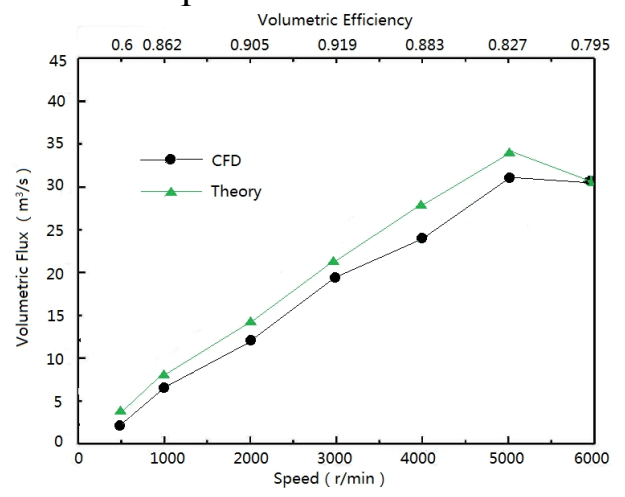

Fig. 6 Flow characteristic curve of new profile oil pump

\section{Pressure distribution}

We see the pressure distribution under design condition in the flow domain of the oil pump (from the Fig.20(a) to (e)). The pressure increases gradually along the radial direction in rotors with a reasonable distribution and smooth transition.

The oil flow through the oil-in cavities to the rotors meshing area and forming a closed region; the pressure around the inlet is lower, with rotors rotating, the closed region will be larger and absorb the oil gradually, the process of oil absorbing will be finished till the closed region runs up to the maximum bulk; while the rotor tooth begin to separate from each other, closed region will be squeezed and the pressure increased, the oil is expelled from the cavities in the end. 


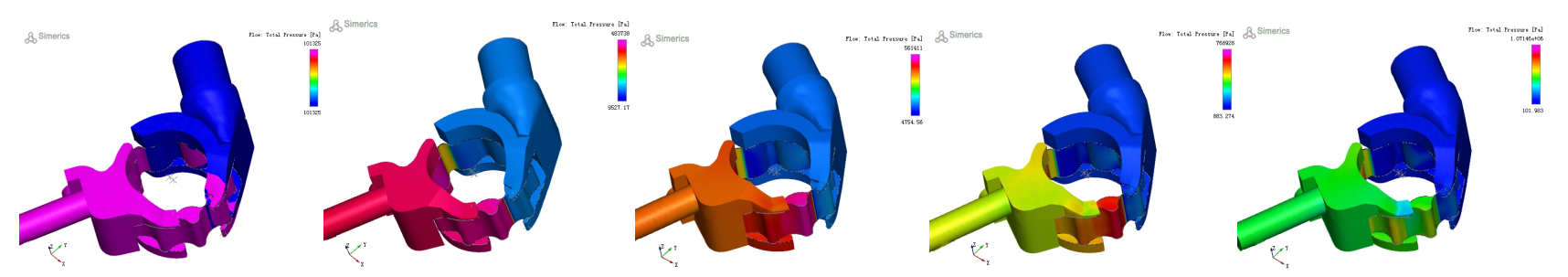

\section{Conclusions}

Based on the analysis of numerical simulation of the inner engaging pump with PumpLinx, the results turn out:

(1) Flow volumetric flux@outlet increased with the rotational speed increases. but decreased at the speed of $4000 \mathrm{r} / \mathrm{min}$ and become unstable. CFD result is close to the theoretical; there are small differences at the high speed, but it is acceptable, the optimized inlet and outlet cavities have a higher volumetric efficiency.

(2) Pressure increases gradually along the radial direction in rotors with a reasonable distribution and smooth transition. However, there is apparent low pressure region generated, cavitation performance needs to be improved.

\section{References}

[1] Yonghua Mao, Guoxiang, Yunping Hu, The Design calculation of a cycloid pump, Journal of Shandong university (engineering science). 10 (2005) 35.

[2] Shengguan Qu, Zishu Sun, The measures to improve the volumetric efficiency of a rotor pump, Journal of Huazhong University ofS cience and Technology. 1 (2011) 29-32

[3] R. Elayaraja, Performance of gerotor oil pump for an automotive engine prediction using CFD analysis and experimental validation, SAE.7 (2009) 28.

[4] Huang Jiangxing, Research for Double-enveloping Cycloid Internal Gear Pump, Machine Tool \& Hydraulics. 4 (2010) 1.

[5] Colin O'Shea, Analysis and optimization of an electrohydraulic power pack for use in a fully active vehicle suspension through the use of computational fluid dynamics, ASME/BATH 2013 Symposium on Fluid Power and Motion Control, Sarasota, Florida, USA, October 6-9, 2013.

[6] Ghazanfarian, Jafar, Analysis of the material and design modifications influence on strength of the cycloidal gear system, Journal of fluids engineering. 16 (2015) 537-546. 